\title{
Recidiva en intestino delgado de tumor de GIST de bajo riesgo. Caso clínico y revisión de la literatura*
}

\author{
Drs. CLAUDIO HEINE T. ${ }^{1}$, PEDRO MEDINA T. ${ }^{1}$, CLAUDIO WAINSTEIN G. ${ }^{1}$, \\ CLAUDIA HURTADO R. ${ }^{2}$, LUIS CONTRERAS M. ${ }^{3}$, FRANCISCO LÓPEZ K. ${ }^{1}$ \\ 1 Unidad de Coloproctología. \\ 2 Laboratorio de Oncología y Genética Molecular. \\ 3 Unidad de Anatomía Patológica. Clínica Las Condes. \\ Santiago, Chile.
}

\begin{abstract}
Recurrence of low risk small bowel GIST tumor. Case report and revision of the literature
\end{abstract}

Gastrointestinal stromal tumors (GIST) are the most common mesenchymatic neoplasm in the digestive tract, representing about $1 \%$ of malignant gastrointestinal lesions. Seventy to eighty percent are benign according to their size and mitotic index as predictors of malignancy. However, in the presence of relapses in patients with low risk according to the current classification and in light of new adjuvant therapies (Imatinib Mesylate), have defined new parameters for estimation of malignant potential.

Key words: Gastrointestinal stromal tumors, GIST, C-KIT, Imatinib Mesylate.

\section{Resumen}

Los tumores del estroma gastrointestinal (GIST) son las neoplasias mesenquemáticas más comunes del tracto digestivo representando cerca del 1\% de las lesiones neoplásicas gastrointestinales. El 70\%-80\% de ellas son benignas siendo clasificados según su tamaño e índice mitótico como predictores de malignidad. Sin embargo, ante la presencia de recidivas en pacientes con bajo riesgo según la clasificación actual y a la luz de nuevas terapias adyuvantes (Mesilato de Imatinib), se han definido nuevos parámetros para su estimación de potencial maligno.

Palabras clave: Tumores del estroma gastrointestinal, GIST, C-KIT, Mesilato de Imatinib.

\section{Introducción}

Los tumores de GIST son neoplasias mesenquimáticas del tracto gastrointestinal que tienen su origen a partir de un precursor de células intersticiales de Cajal. Previamente estos tumores se clasificaron como leiomiomas, leiomiosarcomas o leiomioblastomas y en 1983, Mazur y Clark, fueron los primeros en denominar a estos tumores como neoplasias del tracto gastrointestinal no epiteliales ${ }^{1}$ al encontrar diferencias estructurales e inmunohistoquímicas en comparación con otros tumores del tracto digestivo.

\footnotetext{
*Recibido el 28 de Mayo de 2009 y aceptado para publicación el 2 de Octubre de 2009.

Correspondencia: Dr. Francisco López K.

Lo Fontecilla 147. Santiago, Chile. Fax: 5626104776

E-mail: flopez@clc.cl
} 
Posteriormente en 1998, Hirota y sus colegas, describieron la presencia de mutaciones activantes en el protooncogen C-KIT en pacientes con tumores GIST. Este gen está localizado en el brazo q11-12 del cromosoma 4 y codifica para una proteína receptora de membrana llamada C-KIT o KIT². Esta proteína tiene actividad tirosina-quinasa y cuando existe una mutación que resulta en una ganancia de función de su actividad enzimática, ésta provoca una estimulación independiente, causando una transducción de señales que lleva a una proliferación celular desregulada. Los tumores de GIST presentan distintas localizaciones siendo las más frecuentes los gástricos (40-70\%), de intestino delgado (20-50\%), colorrectales $(5 \%)$ y menos frecuentes en esófago, omento, mesenterio o en retroperitoneo ${ }^{3}$. En general, se presentan como tumores solitarios bien circunscritos rodeados de una fina pseudocápsula ${ }^{4}$. La mayoría de estos tumores son asintomáticos cuando son de pequeño tamaño, sin embargo, pueden diagnosticarse por complicaciones como hemorragias intraabdominales, perforación intestinal o debido al dolor abdominal intenso y recurrente ${ }^{5}$. Al momento del diagnóstico, entre un 10-30\% presentan invasión de tejidos vecinos y cerca de un tercio presenta compromiso metastático siendo el peritoneo y el hígado los sitios mayormente afectados. Además, estos tumores se caracterizan por presentar una alta tasa de recidivas ${ }^{6,7}$. El objetivo de este trabajo es discutir un caso clínico a la luz de nuevos parámetros de clasificación de riesgo en estos tumores.

\section{Caso Clínico}

Paciente mujer de 42 años, quien consulta por cuadro de dolor abdominal difuso de 3 días de evolución, de moderada intensidad y de predominio hipogástrico que en el estudio de imágenes con Tomografía Computada (TC) se aprecia una lesión tumoral dependiente de intestino delgado por lo que se decide cirugía. En la cirugía se identifica una masa de aproximadamente $4 \mathrm{~cm}$ dependiente de íleon a 1 $\mathrm{mt}$ de la válvula ileocecal. Esta formación tumoral se encuentra volvulada y de aspecto necrótico. Se practica una resección intestino delgado parcial con anastomosis latero-lateral. El estudio histopatológico demostró un tumor bien delimitado de 5 $\mathrm{cm}$ de tamaño, moderadamente celular con áreas necróticas y ulceradas que compromete desde la mucosa hasta la subserosa. Este tumor presentó un índice mitótico de hasta 3 mitosis en 50 campos y la inmunohistoquímica reveló reacción positiva para CD-117 (C-KIT) y negativa para CD-34 y Desmina, resultando un diagnóstico de tumor del estroma gastrointestinal de bajo grado. Al evaluarse el caso en comité oncológico y ante las evidencias de su bajo potencial maligno, se decide observación y seguimiento periódico con imágenes. Luego del alta, la paciente se mantuvo en control con tomografía computada de abdomen y pelvis cada 6 meses sin evidencia de recidiva de la enfermedad. Tras 12 meses de su cirugía, la paciente presenta dolor abdominal recurrente en epigastrio e hipocondrio derecho sospechoso de cólico biliar por lo que se realiza una ecografía abdominal que demuestra la presencia de un pólipo vesicular de 8 por $5 \mathrm{~mm}$ de diámetro por lo que se decide someter a una colecistectomía laparoscópica y aprovechar la oportunidad para hacer una revisión del abdomen (second look) por el tumor resecado un año antes.

En el intraoperatorio y durante la exploración general, se observa la presencia de múltiples lesiones polipoídeas implantadas en la superficie serosa del intestino delgado cercanos a la zona de anastomosis y de omento mayor (Figura 1). Una vez efectuada la colecistectomía se procede a efectuar una resección parcial de intestino delgado de aproximadamente $25 \mathrm{~cm}$ realizando una anastomosis latero-lateral terminal funcional. Luego se realiza una resección de omento mayor dependiente de colon transverso donde se aprecian algunas lesiones aisladas. Se cierra la minilaparotomía periumbilical y las incisiones de los trocares sin inconvenientes. La paciente presentó un postoperatorio favorable siendo dada de alta al $4^{\circ}$ día.

El estudio anatomopatológico macroscópico mostró que la pieza de sección intestinal en "Y" presentaba una serosa irregular con presencia de múltiples áreas nodulares (más de 20 con diámetros entre 1 a $7 \mathrm{~mm}$ de eje mayor) (Figura 2) y presencia de áreas granulares en el omento mayor de similares características (de entre 1 a $6 \mathrm{~mm}$ ). El estudio microscópico mostró que la pared del intestino delgado presentaba múltiples formaciones a nivel seroso y subseroso y en algunas ocasiones se encontraba en contacto con la lámina muscular propia constituida por células de aspecto fusado y epiteloídeo con leve a moderada atipia nuclear focal y se observó hasta 3 mitosis por 50 campos (Figura 3). El análisis inmunohistoquímico mostró negatividad para Citoqueratina, Desmina, CD-34 y proteína S100 e intensa positividad para Vimentina y CD-117 (Figura 4). En conclusión, los hallazgos histopatológicos fueron compatibles con metástasis serosas múltiples por tumor de GIST. Luego de su cirugía de recidiva del GIST intestinal, se indicó terapia con Mesilato de Imatinib (Glivec $\left.{ }^{\circledR}\right) 100$ mg. diarios con buena respuesta y tolerancia. Actualmente y luego de seis meses de seguimiento, la paciente se encuentra en buenas condiciones con estudios de imágenes realizados a la fecha que no muestran hallazgos de recurrencia de la enfermedad. 


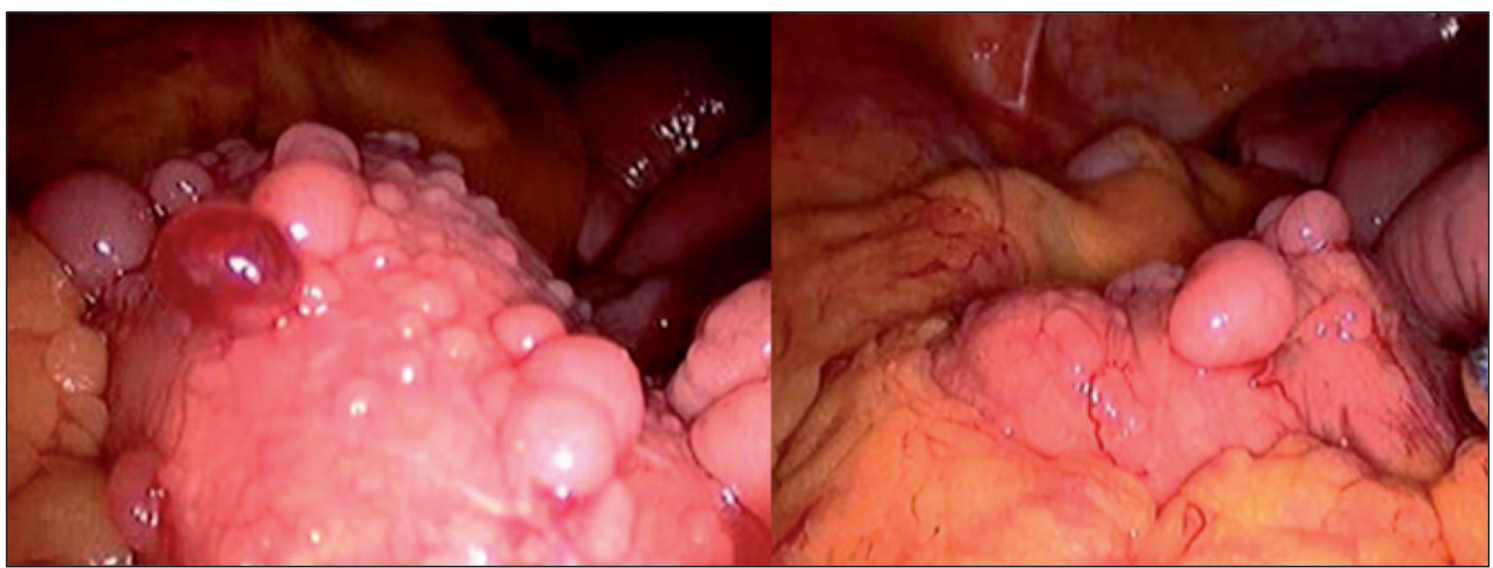

Figura 1. Imágenes laparoscópicas de implantes tumorales en serosa de intestino delgado.

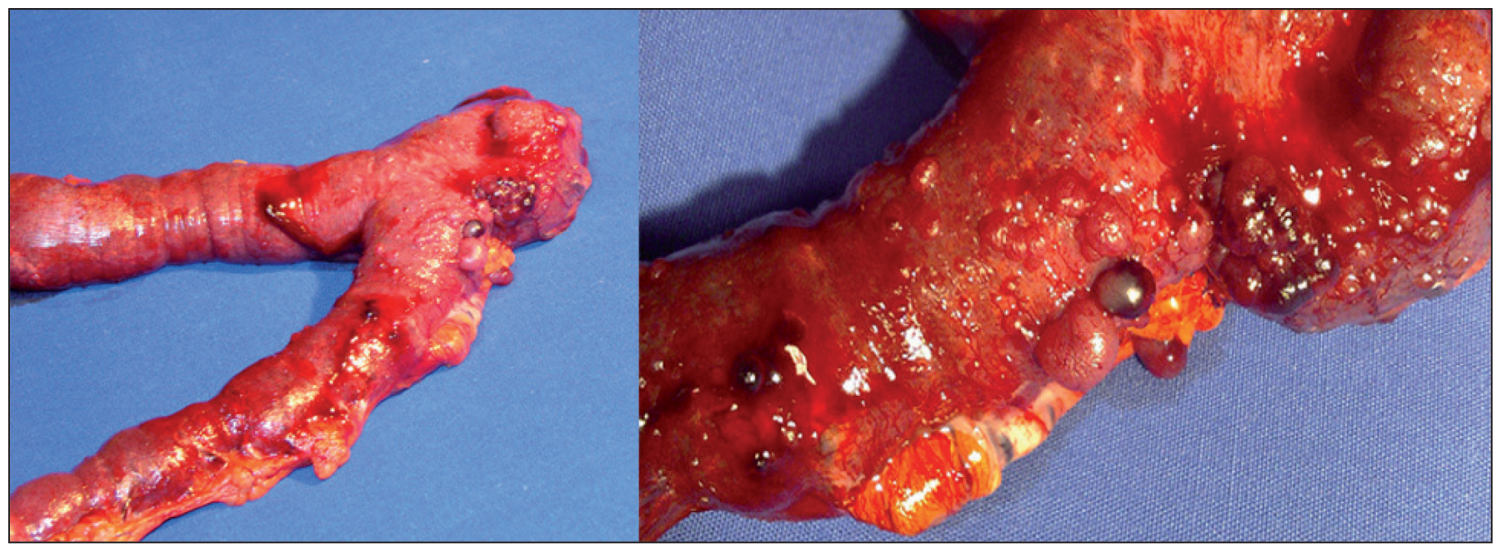

Figura 2. Pieza operatoria (en forma de "Y"). Obsérvese la presencia de lesiones principalmente en la zona de la enteroentero anastomosis.

\section{Discusión}

Los tumores de GIST representan cerca de un 5\% de los tumores mesenquemáticos y cerca del 1\% de las neoplasias malignas gastrointestinales con una incidencia de entre 7 a 20 pacientes por millón. En Chile constituyen de entre el 0,86 al 4\% de las neoplasias gástricas ${ }^{8-10}$. Estos tumores pueden originarse en cualquier segmento del tracto gastrointestinal siendo más frecuentes en el estómago (60-70\%), intestino delgado $(25 \%)$ y en el colon y recto $(10 \%)^{11}$. En el diagnóstico diferencial es importante distinguir los GIST de otros tumores de células fusadas, epiteloides o con patrón mixto, lo que hace del estudio inmunofenotípico una herramienta fundamental. En los estudios de inmunohistoquímica (IHQ), los tumores de GIST se caracterizan por expresar la proteína C-KIT (CD-117) en un $81 \%$ a $100 \%$ diferenciándose de otros tumores como desmoides, leio- miomas, leiomiosarcomas y schwannomas donde la IHQ es siempre negativa; además, se ha observado que entre un $47 \%$ y $70 \%$ de estos tumores también son positivos para CD34; un 5\% son positivos para la proteína $\mathrm{S}-100$ y entre un $1 \%$ a $2 \%$ son positivos para Desmina y Citoqueratina ${ }^{4,12-14}$.

El mejor tratamiento, desde el punto de vista del pronóstico, es la cirugía con resección completa. Aún así, la recurrencia alcanza valores de entre un $27 \%$ a un $84 \%$, con una mortalidad asociada al tumor de entre un $30,8 \%$ a un $68 \%$, siendo menores en los tumores de pequeño tamaño y en los que la resección quirúrgica fue completa ${ }^{15}$. El potencial maligno de los GIST va desde tumores de comportamiento benigno hasta sarcomas agresivos. De estos tumores, un $30 \%$ tienen un comportamiento agresivo, con una alta tasa de recurrencia local, diseminación peritoneal y metástasis hepáticas ${ }^{16}$. En la actualidad, el pronóstico de los pacientes se estra- 


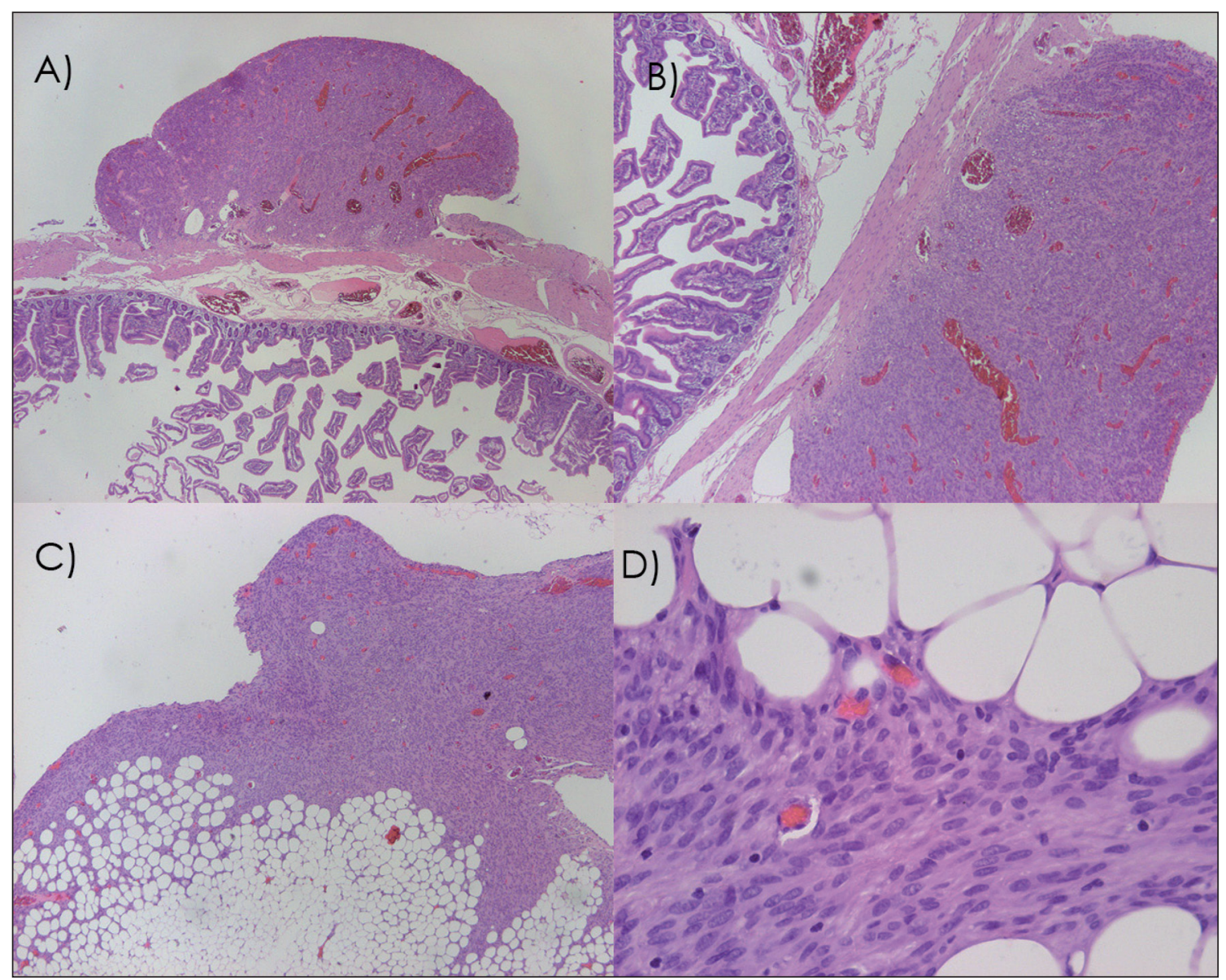

Figura 3. A) y B): Imagen histológica de intestino delgado que presenta formación nodular comprometiendo desde la serosa hasta la muscular propia. C) y D): Tejido fibroadiposo focalmente infiltrado por lesión nodular.

tifica mediante un sistema de clasificación basado en el tamaño tumoral y el índice mitótico descrito por Fletcher el año $2002^{13}$ (Tabla 1). Siguiendo esta clasificación, nuestro caso clínico presentó un índice mitótico de 3 mitosis en 50 campos y un tamaño menor de $5 \mathrm{~cm}$ por lo que el riesgo de recidiva se catalogó como bajo, lo que llevó a la decisión de mantener un control imagenológico cada 6 meses sin necesidad de tratamiento adyuvante. Sin embargo, a pesar de la cirugía con resección completa y márgenes patológicamente negativos, el tumor de GIST recidivó a los 12 meses. Por lo tanto, en la presencia de tumores de GIST de bajo riesgo, la discriminación entre tumor "benigno" o "maligno" no es fácil ya que esta clasificación ampliamente utilizada muchas veces resulta insuficiente. Desde el punto de vista clínico, nueva evidencia revela que el índice mitótico, el tamaño y la localización del tumor serían factores predictivos de recurrencia independientes luego de la resección tumoral $\mathrm{R} 0^{3,7}$, que la presentación sintomática del tumor se asocia con una menor sobrevida libre de enfermedad a 5 años ${ }^{17}$ y que existiría un peor pronóstico en los pacientes con GIST de intestino delgado, colon, recto o mesenterio en comparación con los GIST gástricos ${ }^{18}$. Desde un punto de vista patológico, varios estudios sugieren que el análisis de MIB-1(Ki67) (como marcador celular de proliferación) asociado a la presencia o ausencia de necrosis tumoral según el tamaño tumoral se asociaría a un mayor componente maligno. Esto se debe a que el anticuerpo MIB-1 reconoce el antígeno nuclear Ki-67, que está asociado con la proliferación celular y que se encuentra a lo largo del ciclo celular (fases G1, S, G2, y M), pero no se encuentra en células en reposo (G0) ${ }^{19-21}$ (Tabla 2). Por otro lado, Pérez y cols, han descrito que la expresión de la proteína S-100 como marcador inmunohistoquímico revelaría una menor sobrevida y 


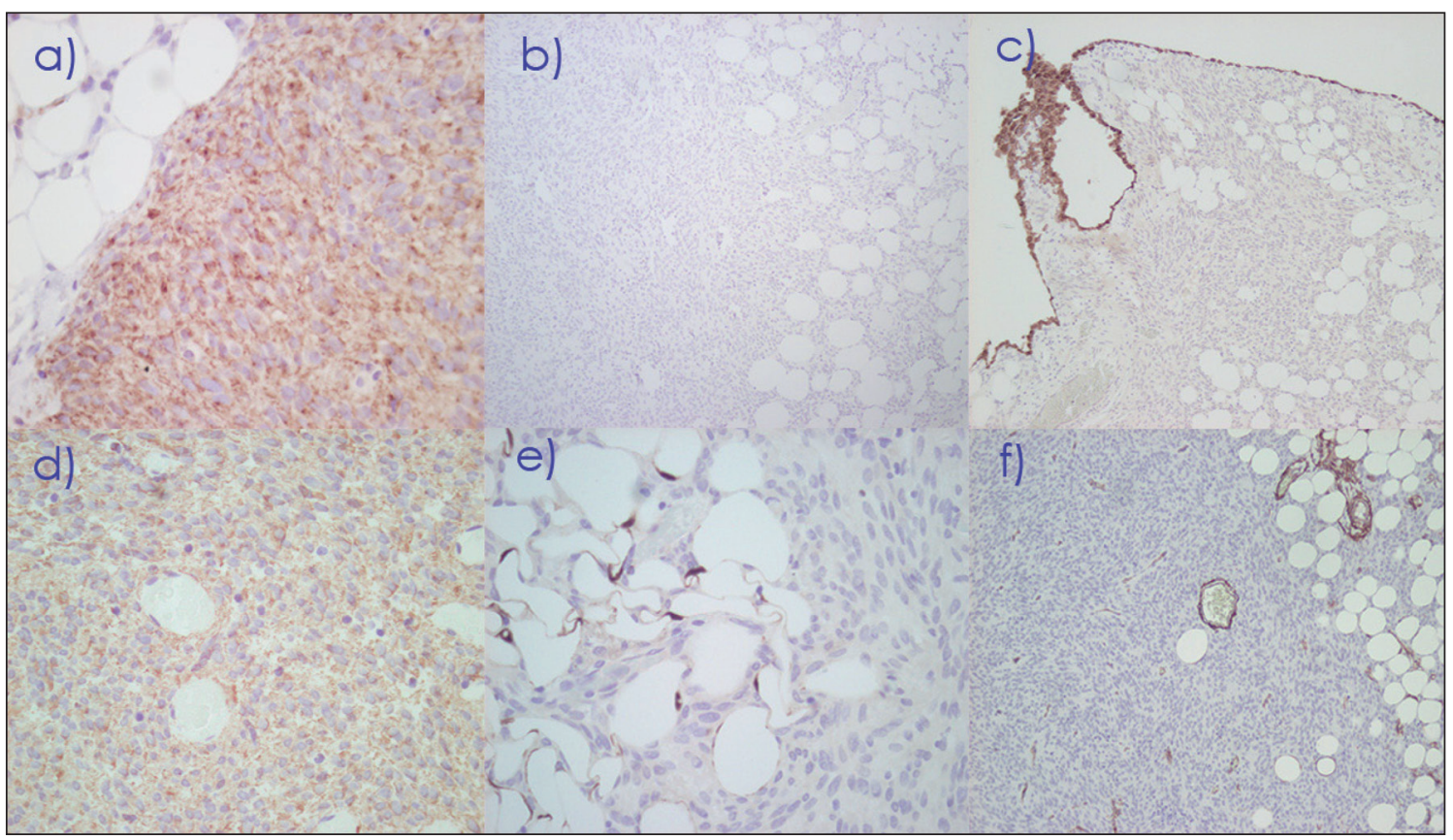

Figura 4. a) Tinción tumoral positiva para CD-117. b) Tinción tumoral negativa para Desmina. c) Tinción tumoral negativa para Citoqueratina. d) Tinción tumoral positiva para Vimentina. e) Tinción tumoral negativa para proteína S100. f) Tinción CD-34 negativa en células tumorales y positiva en vasos sanguíneos.

una tendencia a mayor recurrencia que en los tumores negativos para este marcador ${ }^{22}$. En cuanto a estudios genéticos, la presencia de mutaciones específicas en el gen C-KIT podría predecir una menor sobrevida a 5 años luego de la resección quirúrgica

Tabla 1. Riesgo de recurrencia en tumores de GIST resecables ${ }^{13}$

\begin{tabular}{lll}
\hline Riesgo & Tamaño & Índice Mitótico \\
\hline Muy bajo riesgo & $<2 \mathrm{~cm}$ & $<5$ mitosis en 50 campos \\
Riesgo bajo & $2-5 \mathrm{~cm}$ & $<5$ mitosis en 50 campos \\
Riesgo intermedio & $<5 \mathrm{~cm}$ & $6-10$ mitosis en 50 campos \\
& $5-10 \mathrm{~cm}$ & $<5$ mitosis en 50 campos \\
Riesgo alto & $>10 \mathrm{~cm}$ & Cualquier índice mitótico \\
& Cualquier tamaño & $>10$ mitosis en 50 campos \\
& $>5 \mathrm{~cm}$ & $>5$ mitosis en 50 campos \\
\hline
\end{tabular}

en tumores de GIST localizados ${ }^{23}$. Así mismo, Singer y cols, han demostrado que la presencia de deleciones o inserciones en el exón 11 de este gen dan un valor predictivo independiente de peor sobrevida libre de enfermedad a 5 años ${ }^{24}$. Además, Lasota y cols, demuestran que la presencia de una mutación heterocigota en el exón 11 se asocia con un peor pronóstico con un $85 \%$ de pacientes que desarrollaron metástasis o muerte con un seguimiento promedio de 33,4 meses $^{25}$. Otras variables histopatológicas y biológicas que se encuentran en estudio como factores pronósticos en GIST son el grado de atipia celular, varios factores de crecimiento como IGF1 e IGF2, diversas

Tabla 2. Clasificación alternativa de riesgo en tumores de GIST (19)

\begin{tabular}{|c|c|c|c|}
\hline \multirow[t]{2}{*}{ Índice MIB-1/Ki67 } & \multicolumn{3}{|c|}{ Tamaño tumoral } \\
\hline & $<5 \mathrm{~cm}$ & $5-10 \mathrm{~cm}$ & $>10 \mathrm{~cm}$ \\
\hline MIB- $1<10 \%$ sin evidencia de necrosis histológica & Bajo riesgo & Riesgo intermedio & Alto riesgo \\
\hline MIB-1 > o igual $10 \%$ o evidencia de necrosis histológica & Alto riesgo & Alto riesgo & Alto riesgo \\
\hline
\end{tabular}


proteínas como CD-44, bcl-2, p53, COX-2, p16 (INK4A), p14 (ARF), pRB, MDM2, HIF-1 $\alpha$, c-myc, y otros procesos como ploidía del DNA, actividad de la telomerasa, densidad microvascular y pérdida de la expresión de C-KIT ${ }^{26}$.

El tratamiento específico de los GIST es un derivado de la 2-fenilaminopirimidina con el nombre de Mesilato de Imatinib (Gleevec $\left.{ }^{\circledR}\right)$. Este fármaco actúa compitiendo con la unión de ATP en el dominio intracelular de la quinasa. Esta inhibición competitiva se produce en varias proteínas tirosina quinasas además de C-KIT como PDGFR $\alpha$, PDGFR $\beta$, ABL y ARG. Además se ha demostrado la correlación entre el tipo de mutación en el gen C-KIT y la respuesta a terapia ya que en pacientes con mutación en el exón 11 , un $83 \%$ presentaron buena respuesta y mejor sobrevida general a diferencia de los pacientes con mutación en el exón 9 cuya respuesta fue del $48 \%$ a las dosis estándar y en los pacientes sin mutaciones, la tasa de respuesta fue de un $0 \%{ }^{19,27}$. En la actualidad, las dosis orales fluctúan entre los 100 y los $800 \mathrm{mg} /$ día sin evidencias claras de que la mayor tasa de respuesta o un aumento en la sobrevida se correlacione directamente con la dosis ${ }^{28}$. En Chile, Garrido y cols. muestran una buena respuesta al uso de $400 \mathrm{mg} /$ diarios de Imatinib en una muestra de 4 pacientes con un seguimiento de 40 meses $^{29}$. Cabe señalar que entre un 10\%-20\% de los pacientes tratados presentan resistencia primaria al Imatinib. En el resto de los pacientes, puede aparecer resistencia secundaria, especialmente a partir del segundo año. La resistencia primaria es más común en los GIST con mutación en el exón 9 del C-KIT y en el exón 18 del PDGFR ${ }^{30}$ y la resistencia secundaria se asocia al desarrollo de mutaciones adquiridas como mutaciones en el exón 11 del C-KIT ${ }^{31}$.

\section{Conclusión}

El diagnóstico precoz y la resección completa de los tumores de GIST siguen siendo el pilar fundamental del tratamiento, sin embargo, y en virtud de nueva evidencia, se hace necesaria una nueva clasificación de riesgo para estos pacientes ya que los parámetros utilizados en la actualidad muchas veces resultan insuficientes. En este caso y a la luz de algunos de estos nuevos parámetros de riesgo (ubicación del tumor en intestino delgado, evidencia de necrosis histológica), nuestra clasificación inicial del tumor habría sido de alto grado, lo que nos lleva a considerar el uso de Mesilato de Imatinib al momento del diagnóstico inicial. El inicio de estudios genéticos en pacientes afectados con tumores de GIST, nos podrán orientar acerca del comportamiento tumoral así como la respuesta terapéutica a estos nuevos fármacos.

\section{Referencias}

1. Mazur MT, Clark HB. Gastric stromal tumors: reappraisal of histogenesis. Am J Surg Pathol 1983; 7: 507519.

2. Hirota S, Isoaki K, Moriyama Y, Hashimoto K, Nishida $\mathrm{T}$, Ishiguro $\mathrm{S}$, et al. Gain-of-function mutations of $\mathrm{C}$ KIT in human gastrointestinal stromal tumors. Science 1998; 279: 577-580.

3. De Matteo RP, Lewis JJ, Leung D, Mudan SS, Woodruff JM, Brennan MF. Two hundred gastrointestinal stromal tumors: recurrence patterns and prognostic factors for survival. Ann Surg 2000; 231: 51-58.

4. Pidhorecky I, Cheney RT, Kraybill WG, Gibbs JF. Gastrointestinal stromal tumors: Current diagnosis, biologic behavior and Management. Ann Surg Oncol 2000; 79: 705-712.

5. Catena F, Pasqualine E, Campione O. Gastrointestinal stromal tumors: experience of an emergency surgery department. Dig Surg 2000; 17: 503-507.

6. Rudolph P, Gloeckner K, Parwaresch R, Harms D, Schmidt D. Immunophenotype, proliferation, DNA ploidy, and biological behavior of gastrointestinal stromal tumors: a multivariate clinicopathologic study. Hum Pathol 1998; 29: 791-800.

7. De Matteo RP, Gold JS, Saran L, Gönen M, Liau KH, Maki RG, et al. Tumor mitotic rate, size, and location independentely predict recurrence after resection of primary gastrointestinal stromal tumor. Cancer 2008; 112: 608-615.

8. Llorens P. Resultados del examen masivo del cáncer gástrico en Chile. En: Llorens P, Nakamura K. Diagnóstico y tratamiento de las afecciones gástricas. Tokio: JICA 1995; 143-155.

9. Mucciarini C, Rossi G, Bertolini F, Valli R, Cirilli C, Rashid I, et al. Incidence and clinicopathologic features of gastrointestinal stromal tumors. A population-based study. BMC Cancer 2007; 7: 230.

10. Benavides C, Burmeister R, Apablaza S, García C, Pinedo M. Tumores gástricos estromales. Rev Chil Cir 2002; 54: 44-48.

11. Tran T, Davila JA, El-Serag HB. The epidemiology of malignant gastrointestinal stromal tumors: an analysis of 1458 cases from 1992 to 2000 . Am J Gastroenterology 2005; 100: 162-168.

12. Contreras L. Anatomía patológica de los tumores estromales gastrointestinales (GIST). Rev Méd Clin Condes 2008; 19: 381-384.

13. Fletcher CD, Berman JJ, Corless C, Gorstein F, Lasota J, Longley BJ, et al. Diagnosis of gastrointestinal stromal tumors: a consensus approach. Hum Pathol 2002; 33: 459-465.

14. Hornick JL, Fletcher CD. The role of KIT in the management of patients with gastrointestinal stromal tumors. Hum Pathol 2007; 38: 679-687.

15. Huang CC, Yang CY, Lai IR, Chen CN, Lee PH, Lin 
MT. Gastrointestinal stromal tumor of the small intestine: a clinicopathologic study of 70 cases in the postimatinib era. World J Surg 2009; 33: 828-834.

16. Hueman MT, Schulick RD. Management of gastrointestinal stromal tumours. Surg Clin N Am 2008; 88: 599-614.

17. Hassan I, You YN, Shyyan R, Dozois EJ, Smyrk TC, Okuno SH, et al. Surgically managed gastrointestinal stromal tumors: a comparative and prognostic analysis. Ann Surg Oncol 2008; 15; 52-59.

18. Emory TS, Sobin LH, Lukes L, Lee DH, O’Leary TJ. Prognosis of gastrointestinal smooth-muscle (stromal) tumors: dependence on anatomic site. Am J Surg Pathol 1999; 23: 82-87.

19. Nishida T, Hirota S, Yanagisawa A, Sugino Y, Minami M, Yamamura Y, et al. Clinical practice guidelines for gastrointestinal stromal tumor (GIST) in Japan: English version. Int J Clin Oncol 2008; 13: 416-430.

20. Mochizuki Y, Kodera Y, Ito S, Yamamura Y, Kanemitsu Y, Shimitzu Y, et al. Treatment and risk factors for recurrence after curative resection of gastrointestinal stromal tumors of the stomach. World J Surg 2004; 28: 870-875.

21. Sánchez Hidalgo JM, Muñoz Casares FC, Rufián Peña S, Naranjo Torres A, Ciria Bru R, Briceño Delgado J, y cols. Tumores del estroma gastrointestinal (GIST): factores pronósticos de supervivencia tras citorreducción R0. Rev Esp Enferm Dig 2007; 99: 703-708.

22. Perez D, Demartines N, Meier K, Clavien PA, Jungbluth A, Jaeger D. Protein S100 as prognostic marker for gastrointestinal stromal tumors: a clinicopathological risk factor analysis. J Invest Surg 2007; 20: 181-186.

23. Kim TW, Lee H, Kang YK, Choe MS, Ryu MH, Chang $\mathrm{HM}$, et al. Prognostic significance of C-KIT mutation in localized gastrointestinal stromal tumors. Clin Cancer Res 2004; 10: 3076-3081.

24. Singer S, Rubin BP, Lux ML, Chen CJ, Demetri GD, Fletcher CD, et al. Prognostic value of KIT mutation type, mitotic activity and histologic subtype in gastrointestinal stromal tumours. J Clin Oncol 2002; 20: 3898-3905.

25. Lasota J, vel Dobosz AJ, Wasag B, Wozniak A, Kraszewska E, Michej W, et al. Presence of homozygous KIT exon 11 mutations is strongly associated with malignant clinical behavior in gastrointestinal stromal tumors. Lab Invest 2007; 87: 1029-1041.

26. Joensuu H. Risk stratification of patients diagnosed with gastrointestinal stromal tumor. Hum Pathol 2008; 39: 1411-1419.

27. Heinrich MC, Corless CL, Demetri GD, Blanke CD, von Mehren M, Joensuu H. Kinase mutations and imatinib response in patients with metastatic gastrointestinal stromal tumours. J Clin Oncol 2003; 21: 4342-4349.

28. Demetri GD, von Mehren M, Blanke CD, Van den Abbeele AD, Eisenberg B, Roberts PJ, et al. Efficacy and safety of imatinib mesylate in advanced gastrointestinal stromal tumors. N Engl J Med 2002; 347: 472-480.

29. Garrido M, Moneada M, Tapia G, Méndez G, Galindo $\mathrm{H}$, Huete A, y cols. Efecto de un inhibidor tirosina kinasa (imatinib) en pacientes con tumores estromales gastrointestinales metastásicos. Experiencia preliminar. Rev Méd Chile 2007; 135: 1327-1332.

30. Antonescu C, Bermer P, Guo T, Arkun K, Hom G, Koryotowski B, et al. Acquired resistance to imatinib in gastrointestinal stromal tumours occurs through secondary gene mutation. Clin Cancer Res 2005; 11 : 4182-4190.

31. Van Glabbeke M, Verweij J, Casali PG, Le Cesne A, Hohenberger P, Ray-Coquard I, et al. Initial and late resistance to imatinib in advanced gastrointestinal stromal tumors are predicted by different prognostic factors: a European Organization for Research and Treatment of Cancer-Italian Sarcoma Group-Australasian Gastrointestinal Trials Group study. J Clin Oncol 2005; 23: 5795-5804. 